\title{
The IT-Socket: Model-Based Realisation of the Business and IT Alignment Framework
}

\author{
Robert Woitsch, Wilfrid Utz, and Vedran Hrgovcic \\ BOC Asset Management, Bäckerstraße 5, 1010 Wien, Austria \\ \{Robert.Woitsch,Wilfrid.Utz, Vedran.Hrgovcic\}@boc-eu.com
}

\begin{abstract}
As a result of recent changes in the IT, where the role of IT has shifted from an enabler to an industrial sector in its own right, the necessity to provide framework which would allow seamless alignment of the business and IT rose. The goal was to allow the stakeholders from the business as well as IT perspective to model the requirements for the IT service provisioning using the modeling languages they are familiar with and to apply semantic technologies as a mediator that will allow translation between them. IT-Socket, the model based approach for business and IT alignment, developed in the European Research project - plugIT, aims to realize the vision of businesses "plugging-in" to IT by introducing graphical modeling languages as mediators between the domain experts and IT. The research challenges identified include the ability to perform integration and translation between graphical modeling languages by building the reference ontology, enabling automatic generation of machine interpretable domain ontologies from graphical models and allowing automatic translation between domain ontologies. The results of the application of the ITSocket for business and IT alignment are presented within three use cases: (1) "Certification" of IT infrastructure, (2) "Virtual Organization" by evolving the current service orientation to a higher and more business driven abstraction and (3) "Governance" of IT infrastructure.
\end{abstract}

Keywords: Next Generation Modelling Framework, Knowledge Management, Semantics, IT-Socket, Virtual Organisation, IT-Governance, Certification.

\section{Introduction}

Information technology (IT) has changed during the history in many ways (e.g. Mainframe to Client-Server, SOA, etc) and those changes have influenced the way how we see and use the IT in our everyday work. Currently the IT is influenced by many different factors, the external factors such as rapid changes in the market environment, legal requirements, different regulations as well as the internal factors such as Software as a Service, SOA, etc, which impose the necessity to align the business and IT in order to survive in highly competitive markets. The change in the IT that we are witnessing today is a ground-breaking change from an enabler -supporting business - to an industry on its own - doing business. [13], [11]. 
The change resulted in IT moving away from back-office support toward becoming a core business process (e.g. in branches Airline Transport, Finance, Automotive Industry or Health Care, IT is a strategic factor in business) [6].

Model based approaches are seen as a viable solution to support this change and to allow seamless transition between the enabler to provider, by allowing the alignment based on externalized requirements from both the business and IT perspective.

IT has to support different business coming from various domains and having diverse requirements. The EU research project plugIT - FP7-3ICT-231430 [36] aims to develop a model-based IT-Socket that would allow business to plug-in into the IT. The domain specific scenarios which will evaluate the IT-Socket within the project include (as detailed in [50] and [33]):

- The "Certification Use Case" demonstrates how the alignment between the business area and the IT domain during the certification process for regulations such as SOX, EuroSOX, ITIL ${ }^{\circledR}$, CoBIT $^{\circledR}$, ISO20000 or BASEL II can be established.

- The "Virtual Organization Use Case" demonstrates how virtual organizations can be supported using business driven requirements and semantically described SLA's for intelligent interpretation.

- The "Governance Use Case" demonstrates how intelligent agents are used to identify the IT infrastructure of data centres. Graphical models are regarded as mediators between system administration and an intelligent discovery environment.

The structure of the paper is as follows: after this introductory section a brief overview on the idea and background of the IT-Socket is presented - focusing on the related work and the analysis of the IT-Socket. Following section is dedicated to the model based realization approach of the IT-Socket. Last section summaries the work presented and provides overview on planned future work.

\section{Idea and Definition of the IT-Socket}

The standardisation and industrialisation of everyday work which affected more and more business sectors in last decades did not make halt before the IT. The trend being currently observed, when analysing the industrialisation phenomenon in the IT, can be easily compared with the industrialisation of the electricity where electric power is provided and consumed via power sockets [15], [25].

Building on this observation the plugIT [36] defined a challenge to realise an ITSocket similar to the aforementioned electric power socket, providing a standardized and industrialised access to consumers of the IT services.

The first step in order to realize the IT-Socket in plugIT was the research on related approaches (as depicted in section 2.1) that range from formal, to unstructured and up to intuitive mechanisms in order to define the modelling framework that will be used for modelling and configuring domain specific IT-Socket.

It is assumed that expert knowledge from both parts - business and IT perspective - can be externalized, formalised and used to support the business and IT alignment by applying semantic technologies for integration, translation and transformation of the externalized knowledge. 


\subsection{Related Work}

The importance of the business and IT alignment has been recognized as a significant part of the enterprise life cycle by various actors coming from different areas of business and IT world.

Due to this diversification of research we witness different approaches on business and IT alignment which focus mainly on technical transformation, like described in [39] and [9], or on the other hand involving also the business aspects as elaborated in [41] and [4].

The business perspective - the set of initiatives, vendors and guidelines has been described in [43] - as one of the parts of the alignment has been underestimated in the SOA based approaches. A survey of 175 research papers about SOA from 2000 to 2008 outlines this statement [46].

The goal of solving the issue and integrating the aforementioned business perspective is done through provision of the detailed description of the alignment between business requirement specification and IT and it is fulfilled by externalization of participants' knowledge within the alignment process. The model-based approach applied in the plugIT provides a way to conceptually link the business requirements and IT [47], [21].

By applying the model-based approach the alignment is described by models representing the initial step of formalization. The key challenge is the integration of different modeling languages that are used to describe the business and IT perspective.

The alignment of the business and IT can be achieved by following different approaches and thus yielding different results. Some of the approaches in this area that have been evaluated by plugIT include:

- The Formal approach - is following the strategy of applying mathematical models in order to identify best-suited IT products for a specific business scenario. An example of the formal approach for the alignment include IT-Portfolio Management from Zimmermann [49] and the identification of services based on business process model analysis from Esswein et al, [12].

- The Heuristic approach - applies the similar approach as the previously mentioned Formal approach, but it reduces the formalisms details level. Prominent examples include the questionnaires provided by Technology Evaluation Centre [42] in order to support the end user to formulate the business request, the model driven business application system development [22] or the SOAM framework [34].

- The Informal approach - is probably the most common approach found today. It involves usage of Request for Information (RFI), Request for Quotation (RFQ), Request for Tender (RFT) and Request for Proposal (RFP) in order to align the business requirements and provided IT services. An overview on this approach can be found in [28] and [38].

- The Intuitive Alignment approach - is a human-driven approach for alignment, which relies on initiative human competence to align business requirements and existing IT products. The advantage is that it includes experts in the alignment process and possible disadvantage could be that it relies solely on the expertise of the involved persons. 
The alignment followed by the IT-Socket approach does not follow any specific alignment approaches nor tries to create a standard business and IT alignment approach but introduces a holistic model-based framework that can be used in different ways to ensure business and IT alignment.

\subsection{The Analysis of the IT-Socket}

In order to address the definition and analysis of the IT-Socket the first task was to analyse both business and IT perspective and to identify the relevant parts playing major role in the business and IT alignment. The starting point to identify these requirements were the business processes for the business perspective and IT products - the commercially exploitable bundle of IT services - for the IT perspective. The process of analysing the IT-Socket was based on three aspects:

- Interviewing experts in order to externalize the required knowledge in interviews and number of modelling sessions. These initial tasks were carried out with the plugIT use case partners - iTG ${ }^{1}$, HLRS $^{2}$ and CINECA ${ }^{3}$

- Complement experiments at universities in order to validate the results of the interviews and modelling sessions against the visionary challenge of the IT-Socket

- Extensive survey in the literature [3], [7], [10], [14], [23], [29], [31], [40] and [48]

The result of the analysis, as shown in Fig 1, was the definition of three aspects for both the business and IT perspective: the competence aspects - providing the human knowledge, technical aspects - consisting of software, hardware and IT infrastructure and organisational aspects - taking over responsibility for parts of the IT-Infrastructure. They are divided on the perspective basis and include:

- Competence requirement to correctly specify the IT products.

- Technical requirements of the IT products.

- Organisational requirements to correctly specify the IT products,

for the business perspective and

- Competence provision of an IT service.

- Technical provision of an IT service.

- Organisational provision of an IT service.

for the IT perspective.

The IT services are defined in following pillars [50]:

- IT services in form of competence provision like helpdesks, training or consulting,

- IT services in form of technical provision like applications, middleware or housing as well as

\footnotetext{
${ }^{1}$ ITG, Innovation Technology Group SA, http://www.itg.pl

${ }^{2}$ HLRS, High Performance Computing Center Stuttgart, http://www.hlrs.de

${ }^{3}$ CINECA, Consorzio Interuniversitario, http://www.cineca.it
} 


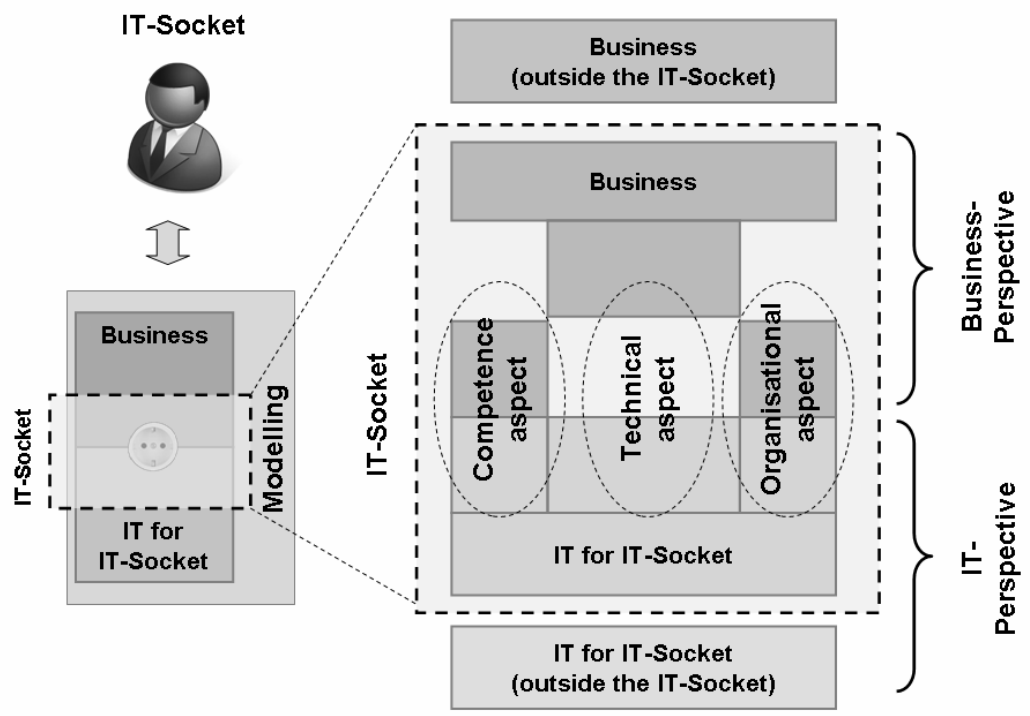

Fig. 1. Identified aspects of the IT-Socket, [35]

- IT services in form of organizational provision like maintenance processes, user administration or infrastructure monitoring.

Another important factor in the analysis of the IT-Socket that has to be taken into account is the so-called abstraction layer of the IT-Services. This simplified means that IT services like data storage or server housing are considered to have lower abstraction than services providing for example ERP functionalities.

Abstraction layer signals the distance of the IT service to the specific activity of the business process that is consuming the IT-Service [50]. More detailed definition of these abstraction layers is currently not possible as different application domains may have different abstraction levels - see [44], [18], and different implementation at the client site (plugIT Use Cases).

This results in the issue that the IT-Socket has to support service provision considering different abstraction layers and different classification of the abstraction itself.

The answer to this question is the introduction of the model based framework which is easily adaptable by the service provider in order to comply with the imposed requirements of the end user (on any abstraction layer), thus being generic in such way to support the requirements (in any specific instantiation of the IT-Socket) and still be capable of handling the available IT services.

The alignment between the requests and the provisioning, required to allow the usage of the presented framework, is assured through formalizing the request performed by the end user so that following conditions are satisfied [50]: 
- the correct abstraction layer for the specified IT service can be easily identified,

- the required IT service parameters are explicitly described to allow the identification of the most appropriate IT service for the specific business use case and

- the appropriate product framework in terms of legal aspects, responsibility, additional services like training, service, or helpdesk as well as the financial conditions can be identified.

ACS [1], CISR [8], ISACA [16], ITGI [17] and OCG [32] provide different alignment approaches, whereas [45] provides an overview on 17 different methods to reach the desired goals. The selection of one of the approaches within the model based framework presented here depends on complexity of the IT product, the competence, the organizational culture and the like.

\section{The Model-Based Realization of the IT-Socket}

As outlined in the related work section of the paper, the model based realization of the alignment framework has to deal with different alignment approaches. The model based approach builds on the foundation that the relevant knowledge, both for the business and IT part, can be externalized in both semi-formal and formal way using graphical models. The ability to translate between the different languages (one of the research challenges defined in section 3.2) allows both stakeholders, the business and IT managers to use the languages they are familiar (e.g. BPMN, UML, etc) with in order to describe the requirements for a specific use case.

\subsection{The Six Elements of the IT-Socket}

Fig. 2 depicts the two perspectives: business - the "plug" containing two elements and IT - the "IT-Socket" and corresponding six elements of the IT-Socket. The first two elements forming the "plug" include:

- Business - this is expressed using models in order to graphically describe such aspects as knowledge, business processes, business rules, etc. The model based modelling framework of the IT-Sockets takes into account all business aspects that are relevant for the IT-Socket and specific use case (e.g. business processes requiring alignment with the underlying IT) and those that are not directly relevant for the IT-Socket (e.g. business strategies that do not directly influence the business and IT alignment),

- Business Requirements - this is applied in the definition of the T services used. Based on the selected approach the requirement specification may be expressed in mathematical formulas, fulfilled questionnaires and the like - as described in section 2.1

The second part depicted in the Fig 2 is the IT-socket consisting out of six elements, where first four describe the IT perspective and last two are describing the business perspective of the IT-Socket. Starting from bottom up following elements are available: 


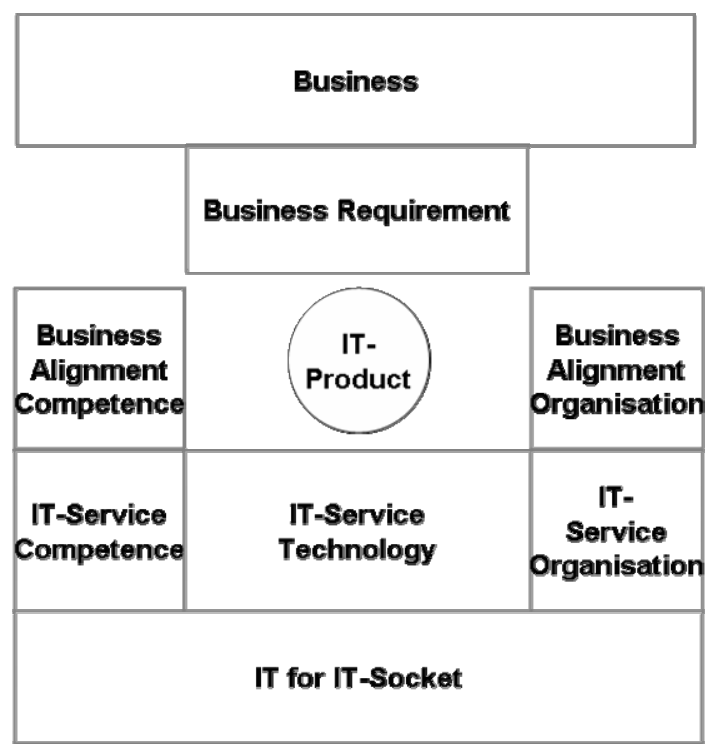

Fig. 2. The IT-Socket framework, [35]

- IT for the IT Socket - this element includes basic items required to provide IT services to end users. This includes software, hardware and corresponding infrastructure. This element, depending on the level of detail in which IT governance is applied in the alignment process, may be considered as not relevant for the IT-Socket.

- IT Service Technology - this element represent all services (e.g. software based services) that are offered to the end users having different abstraction layers as defined in section 2.2

- IT Service Competence - this element represents and describes all services in terms of competence provision. These services include such functionalities like helpdesk, training, etc., which may not be seen as technical services but are nevertheless included as they assure the deployment of technical services.

- IT Service Organisation - organisational IT services handle such aspects for which the provider of the IT service is also responsible, e.g. backup, maintenance, test, etc.

- Business Alignment Competence - the goal within this element is to explicitly define the skills required to execute the select approach and perform the business and IT alignment.

- Business Alignment Organisation - defines the processes that are performed during the alignment.

All described elements may use different languages and each of the applied modelling languages can have different formal expressions. This manifests in having collections of different models designed using different languages and having different levels of formality. 


\subsection{The Semantic within the IT-Socket}

The alignment scenario presented in the paper requires IT-Socket to be able to handle different modelling languages describing different perspectives, aspects and formalisation levels. This involves the necessity to utilize the semantic technologies in order to act as a mediator between the (1) modelling languages and (2) business and IT models. The major task is to guarantee that imposed service requirements by the end user which are designed in the language that end user is familiar with are understood by IT service providers which may use different modelling languages with different formal expressiveness.

Models describing one of the aforementioned elements of the IT-Socket (section 3.1) may, based on the alignment procedures requiring different formalisation levels, be formal, semi-formal or unstructured. So in one use case stakeholders may be confronted on one hand with text documents and on other hand with mathematical definitions. In order to be able to mediate between these different definitions, semantic technology is used to translate between the used modelling languages, namely it is used to provide an integrated and coherent view of data stored in multiple, heterogeneous information sources. The integration of the available information is an important research field and have been prominently represented in recent research activities.

Different forms of integration can be distinguished [50]:

- Schema integration: Design a global unified schema

- Data Integration: Take into account both schema and actual data

- Semantic integration: Take into account ontologies, schemata and data which can be structured, unstructured or semi-structured

Three dimensions of the semantic integration techniques are applied in the IT-Socket [50]:

- Mapping discovery: Given two ontologies/schemas, how do we find similarities between them, determine which concepts and properties represent similar notions, and the like.

- Declarative formal representations of mappings: Given two ontologies, how do we represent the mappings between them to enable reasoning with mappings?

- Reasoning with mappings: Once the mappings are defined, what do we do with them, what types of reasoning are involved?

Based on aforementioned semantic integration techniques we try to address following five research challenges (as depicted in Fig. 3. and detailed in [50]):

- $\mathrm{RC} 1$ - The first research challenge focuses on the development of the modelling language ontologies (marked as MLO in Fig.3). The MLO is created by transforming the expressions of the graphical modelling languages into a well formed ontology used to represent the modelling principles of the modelling language in question. Besides the meaning that is extracted from the graphical elements, a textual description has to be exposed too in order to allow interoperability. This approach is applicable to any modelling language; example would be to specify the elements of UML or BPMN as ontology - see [19]. 


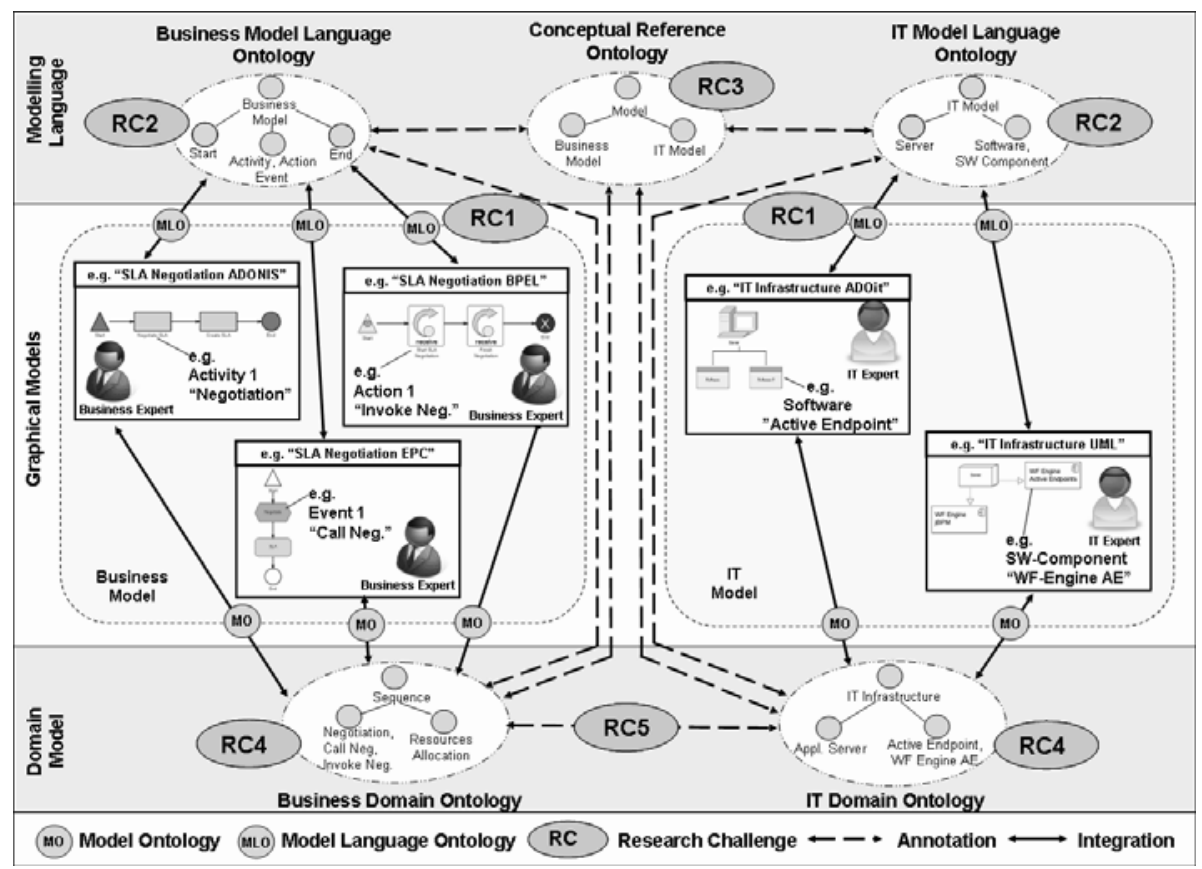

Fig. 3. An overview on the research challenges indentified in the project in order to realize the IT-Socket, [50]

- RC2 - The second research challenge is dedicated to the integration and translation of the modelling languages used by different stakeholders that will use the IT-Socket. In order to allow the integration and translation it is necessary to take into account syntactic and semantic layers (as elaborated in [24] and [30]). The syntactic layer is provided as a result of the $\mathrm{RC} 1$. The representative ontology is used in order to make possible the mapping between the source modelling languages (stakeholder describing the scenario) and target modelling languages (stakeholder as service provider). Such an approach is elaborated in [5], [26], [27] and [37]

- RC3 - The third indentified research challenge is focused on developing the reference ontologies for the modelling languages used in the plugIT with the goal to analyse the ability to use such ontology to explicitly transform between different modelling languages. This will be handled on the meta-level. The meta meta level (comp. [20]) for modelling languages - as used in ADONIS, MOF, etc, - describes the generic elements of the modelling languages (comp. [24], [30]). The meta level used in the RC3 is in the example of modelling languages the description of the aforementioned elements as derivations of the meta meta element, e.g. activity, process start, etc. The ontology developed in the RC3, the Conceptual Reference Ontology (marked with CRO in Fig. 3) is based on the meta meta level but requires parts of the meta level in order to be used for mapping. 
- $\mathrm{RC} 4$ - The fourth research challenge is tackling the issue of automatic generation of the machine readable domain specific ontologies out of the graphical models. Such an ontology (the modelling ontology - marked with MO) is generated out of graphic model where each model was annotated with the previously available MLO. By doing this the machine-interpretability is assured and on the other hand these mechanisms ensure continuous evolution of the domain ontology and enable cooperative modelling by different domain experts using different tools.

- RC5 - The fifth research challenge is dedicated to the translation between domain specific ontologies using the MLO and CRO ontologies as a bridge to map the ontology concepts. This provides the functionality to the stakeholder to use their own language (e.g. business manager using BPMN and IT Manager using UML) to define and model the requirements for a specific case and to present them to the counterpart in their own language as mapping and translation will be handled by the IT-Socket.

\section{Conclusion}

The work presented here introduced a business and IT alignment approach applying the model-based IT-Socket. Currently this approach is being implemented by the consortium of the EU research project plugIT. As the project is currently in its early phase only the initial results such as definition of the research challenges as well as the analysis of the IT-Socket are presented.

Further work is separated in two parts, first one focusing on stronger involvement of semantic technologies - through application of semantic and meta modelling matching patterns [24] - conceptual integration, development of the Next Generation Modelling Framework and application of the abstract workflows in order to support the execution of the IT-Socket aligned processes - the technical integration of the framework and on the other hand the evaluation of the IT-Socket in the three end user use cases.

Following the Open Model paradigm ${ }^{4}$, The Next Generation Modelling Framework will be available as a public service to attract the community to use the webmodelling platform to design and the execution environment to deploy the IT-Socket.

Acknowledgments. The authors would like to thank the members of Elsag Datamat, Consorzio Interuniversitario CINECA, Innovation Technology Group SA and University of Stuttgart, High Performance Computer Centre as members of the plugIT consortium for the cooperation of this publication.

The authors especially acknowledge the contribution from Prof. Dr. Dimitris Karagiannis (University of Vienna), Prof. Dr. Plexousakis (Foundation for Research and Technology-Hellas) and Prof. Dr. Hinkelmann (Fachhochschule Nordwestschweiz) for their contribution to this publication.

\footnotetext{
${ }^{4}$ www.openmodels.at
} 


\section{References}

1. ACS. Governance of Information and Communication Technology Committee (2009), http: / / www . acs . org.au / governance/ (accessed May 29, 2009)

2. Andrea, J.: An Agile Request For Proposal (RFP) Process. In: ADC 2003: Proceedings of the Conference on Agile Development. IEEE Computer Society, Washington (2003)

3. Bloomberg, J.: SOA Governance - IT Governance in the context of service orientation (2004),

http: / / www . zapthink. com/report.html ?id=ZAPFLASH-10272004 (accessed May 29, 2009)

4. BREIN. Business objective driven REliable and Intelligent grids for real busiNess (2009), http: / / www . eu-brein. com/ (accessed May 29, 2009)

5. Calvanese, D., Giacomo, G., Lenzerini, M., Nardi, D., Rosati, R.: Description Logic Framework for Information Integration. In: International Conference on Knowledge Representation and Reasoning (KR), pp. 2-13 (1998)

6. Carr, N.G.: IT doesn't matter, HBR, BCG Analysis (May 2003)

7. Charlsworth, I., Davis, D.: Getting to grips with SOA governance. Ovum Report \#040077, 2006-23 (2006)

8. CISR. Center for Information Systems Research (2009), http: / / mitsloan.mit.edu/cisr/research.php (accessed May 29, 2009)

9. COMPAS. Compliance-driven Models, Languages, and Architectures for Services (2009), http: / / www. compas-ict. eu / (accessed May 29, 2009

10. Dostal, W., Jeckle, M., Melzer, I., Zengler, B.: Service-orientierte Architekturen mit Web Services: Konzepte - Standards - Praxis, 2nd edn. Spekturm Akademischer Verlag, München (2007)

11. EITO. In cooperation with IDC, European Information Technology Observation (2006), http: / / www. eito. com (access: 04.04.2008)

12. Esswein, W., Weller, J., Stark, J., Juhrisch, M.: Identifikation von Sevices aus Geschäftsprozessmodellen durch automatisierte Modell analyse. In: Proceedings of WI 2009, pp. 513-522 (2009),

http: //www.dke.univie.ac.at/wi2009/Tagungsband_8f9643f/

Band2 .pdf (accessed May 15, 2009)

13. Forrester Research, European IT Services (2007),

http: / /www. forrester.com/Research/Document/Excerpt/

0,7211, 38932, 00 .html (accessed: April 4, 2009)

14. Hedin, M.: SOA-Driven Organizational Change Management: A Market Trends and Vendor Landscape Analysis of Major Service Players to Address this Emerging Opportunity. IDC Competitive Analysis \#204727 (2006)

15. Hochstein, A., Ebert, N., Uebernickel, F., Brenner, W.: IT-Industrialisierung: Was ist das? Computerwoche, 15 (2007)

16. ISACA. Information Systems Audit and Control Association (2009), http: / /www. isaca.org/ (accessed May 29, 2009)

17. ITGI. IT Governance Institute (2009), http://www.itgi.org (accessed May 29, 2009)

18. ITIL. IT Infrastructure Library (2009),

http://www.itil-officialsite.com/home/home.asp (accessed May 29, 2009) 
19. Kappel, G., Kapsammer, E., Kargl, H., Kramler, G., Reiter, T., Retschitzegger, W., Schwinger, W., Wimmer, M.: On Models and Ontologies - A Layered Approach for Model-based Tool Integration. In: Mayr, H.C., Breu, R. (eds.) Modellierung 2006, pp. $11-$ 27 (2006)

20. Karagiannis, D., Höfferer, P.: Metamodels in Action: An overview. In: Filipe, J., Shishkov, B., Helfert, M. (eds.) ICSOFT 2006 - First International Conference on Software and Data Technologies:IS27-36. Insticc Press, Setúbal (2006)

21. Karagiannis, D., Utz, W., Woitsch, R., Eichner, H.: BPM4SOA Business Process Models for Semantic Service-Oriented Infrastructures. In: eChallenges e-2008. IOS Press, Stockholm (2008)

22. Kätker, S., Patig, S.: Model-Driven Development of Service-Oriented Busienss Application Systems. In: Proceeding of Wirtschaftsinformatik 2009, pp. 171-180 (2009), http: //www.dke.univie.ac.at/wi2009/Tagungsband_8f9643f/ Band1.pdf (accessed May 29, 2009)

23. Kohnke, O., Scheffler, T., Hock, C.: SOA-Governance - Ein Ansatz zum Management serviceorientierter Architekturen. Wirtschaftsinfor 50(5), 408-412 (2008)

24. Kühn, H.: Methodenintegration im Business Engineering, PhD Thesis (2004)

25. Lamberti, H.-J.: Banken Technologie als Schlüssel für die Bank der Zukunft. Leistung aus Leidenschaft Deutsche Bank (2009),

http: / /wi2009.at/fileadmin/templates/downloads /

2009_0227_Lamberti_WI2009_Wien_Versand.pdf (accessed May 29, 2009)

26. Lembo, D., Lenzerini, M., Rosati, R.: Review on models and systems for information integration, Technical Report, Universita di Roma "La Sapienza" (2002)

27. Manakanatas, D., Plexousakis, D.: A Tool for Semi-Automated Semantic Schema Mapping: Design and Implementation. In: Proceedings of the Int. Workshop on Data Integration and the Semantic Web (DISWeb 2006), pp. 290-306 (2006)

28. Mhay, S.: Request for. Procurement Processes, RFT RFQ RFP RFI (2009), http: / / www. negotiations.com/articles/procurement-terms / (accessed May 29, 2009)

29. Mitra, P., Noy, N., Jaiswal, A.R.: OMEN: A Probabilistic Ontology Mapping Tool. In: Gil, Y., Motta, E., Benjamins, V.R., Musen, M.A. (eds.) ISWC 2005. LNCS, vol. 3729, pp. 537-547. Springer, Heidelberg (2005)

30. Murzek, M.: The Model Morphing Approach - Horizontal Transformation of Business Process Models, PhD Thesis (2008)

31. OASIS. OASIS (2009), http: / /www . oasis-open. org (accessed May 29, 2009)

32. OCG. AK IT-Governance - Aufgaben und Ziele (2009), http: / /www.ocg.at/ak/governance/index.html (accessed May 29, 2009)

33. plugIT D 5.1: Use Case and Evaluation Criteria (2009), http://plug-it-project.org

34. Offmann, P.: SOAM - Eine Methode zur Konzeption betrieblicher Software mit einer Serviceorientierten Architektur. Wirtschaftsinformatik 50(6), 461-471 (2008)

35. plugIT D2.1: Use Case Analysis and Evaluation Criteria Specification (2009), http: / /plug-it-project.eu/CMS / ADOwebCMS / upload/ plugIT_D2.1_Use_Case_Description_Evaluation.pdf (accessed May 29, 2009)

36. plugIT. EU-Project FP7-3ICT-231430, plugIT HomePage (2009), http: / / www.plug-it.org (accessed May 29, 2009)

37. Rahm, E., Bernstein, P.A.: A Survey of Approaches to Automatic Schema Matching. The VLDB Journal 10(4), 334-350 (2001) 
38. Shawn, J.: Beyond the Template: Writing a RFP that Works (2009), http: / /www.sourcingmag.com/content/c070228a.asp (accessed May 29, 2009)

39. SOA4ALL. Service Oriented Architectures for All (2009), http: / /www . soa4all . eu (accessed May 29, 2009)

40. Strohm, O., Ulich, E.: Unternehmen arbeitspsychologisch bewerten: ein Mehr-EbenenAnsatz unter besonderer Berücksichtigung von Mensch, Technik und Organisation. Hochschulverlag AG an der ETH, Zürich (1997)

41. SUPER. Semantics Utilized for Process Management within and between Enterprises (2009), http: / /www. ip-super.org/ (accessed May 29, 2009)

42. TEC. Technology Evaluation Centers (2009), http: / / www. technologyevaluation. com (accessed May 29, 2009)

43. Teubner, A., Feller, T.: Governance und Compliance. Wirtschaftsinformatik 50(5), 400407 (2008)

44. The Open Group. TOGAF Version 9 (2009), http: / / www . opengroup.org/togaf / (accessed May 29, 2009)

45. Thomas, O., Leyking, K., Scheid, M.: Vorgehensmodelle zur Entwicklung Serviceorientierter Softwaresysteme. In: Proceedings of Wirtschaftsinformatik 2009, pp. 181-190 (2009), http://www.dke.univie.ac.at/wi2009/Tagungsband_8f9643f/ Band1pdf (accessed May 29, 2009)

46. Viering, G., Legner, C., Ahlemann, F.: The (Lacking) Business Perspective on SOA Critical Themes in SOA Research. In: Proceedings of Wirtschaftsinformatik 2009, pp. 4554 (2009), http://www.dke.univie.ac.at/wi2009/Tagungsband_8f9643f/ Band1 .pdf (accessed May 29, 2009)

47. Willcocks, L.P., Lacity, M.C.: Global Sourcing of Business \& IT Services. Palgrave Macmillan, New York (2006)

48. Windley, P.J.: Teaming up for SOA. InfoWorld (2007), http: / / www. infoworld.com/t/architecture/teaming-soa-620 (accessed May 29, 2009)

49. Zimmermann, S.: Governance im IT-Portfoliomanagement - Ein Ansatz zur Berücksichtigung von Strategic Alignment bei der Bewertung von IT. Wirtschaftsinformatik 5, 357$365(2008)$

50. Woitsch, R., Karagiannis, D., Plexousakis, D., Hinkelmann, K.: Business and ITAlignment: the IT-Socket. Elektrotechnik \& Informationstechnik, 7-8 (2009) 